\title{
Correction to: Beyond Official Development Assistance
}

\section{Correction to:}

L. Jiang, Beyond Official Development Assistance, Governing China in the 21st Century, https://doi.org/10.1007/978-981-32-9507-0

The original version of the book frontmatter was published with old ISSN, which has now been updated with the new ISSN. The correction to the book has been updated with the changes.

The updated version of the book can be found at https://doi.org/10.1007/978-981-32-9507-0

(C) The Author(s) 2020

L. Jiang, Beyond Official Development Assistance, Governing China in the 21 st Century, https://doi.org/10.1007/978-981-32-9507-0_7 